\title{
Inverse Dynamic Control of a 2-DOF Driving Simulator Platform
}

\section{Julio Javier Avalos García ${ }^{1}$, Eduardo Izaguirre Castellanos ${ }^{2}$, Luis Hernández Santana ${ }^{3}$}

\begin{abstract}
${ }^{1}$ Docente - Grupo de Automatización Robótica y Percepción (GARP). Departamento de Automática. Facultad de Igeniería Eléctrica Universidad Central "Marta Abreu" de Las Villas (UCLV), Cuba.

Email: javalos@uclv.cu

Received: November $28^{\text {th }}, 2017$

Accepted: January $14^{\text {th }}, 2018$

Published: March $30^{\text {th }}, 2018$

Copyright (C2016 by authors and Institute of Technology Galileo of Amazon (ITEGAM). This work is licensed under the Creative Commons Attribution International License (CC BY 4.0).

http://creativecommons.org/licenses/by/4.0/ (c) (i) (3) Open Aceets

ABSTRACT

Dynamic modelling is the basic element for controller design of robotics' mechanisms. In this paper, a dynamic equation of a 2-DOF Parallel Driving Simulator Platform has been derived by the Newton-Euler formulation. The proposed model leads to decoupling dynamic characteristics, in which the complexity of the controller design can be significantly reduced. A model-based Computed-Torque Control strategy with a PD Controller in the Cartesian space is implemented in order to obtain the desired performance of the system. The Cartesian control performs an accurate positioning of the end effector, according to the desired task-space specifications. The proposed control strategy is simulated using the MATLAB software package, where simulation results prove that the designed control motion simulator is adequate to perform different driving situations according with the performance specifications of the system.
\end{abstract}

Keywords: 2-DOF, Cartesian space, Computed Torque, Parallel Robot, PD, Platform.

\section{Control por Modelo Dinámico Inverso de Simulador de} Conducción de 2 Grados de Libertad

\section{RESUMEN}

El modelado dinámico de mecanismos robóticos constituye la base para el diseño del Control. En este trabajo se halla una ecuación dinámica de una Plataforma de Simulación de Conducción basada en un mecanismo Paralelo de 2 GDL usando el método de Newton-Euler. El modelo propuesto presenta características dinámicas desacopladas, por lo que se reduce considerablemente la complejidad al diseñar el Controlador. Para lograr el desempeño deseado del sistema se implementa la estrategia de Control por Modelo Dinámico Inverso acompañada por un Controlador PD en el espacio Cartesiano. El control Cartesiano brinda un correcto posicionamiento del elemento final de acuerdo con las especificaciones deseadas en el Espacio de Tareas. La estrategia de control propuesta es simulada en MATLAB, donde los resultados muestran que el diseño del Control para el Simulador es adecuado al desarrollar diferentes situaciones de conducción según las especificaciones físicas del sistema.

Palabras Claves: 2 GDL, Espacio Cartesiano, Control por Modelo Dinámico Inverso, Robot paralelo, PD, Plataforma.

\section{INTRODUCCIÓN}

Generalmente las plataformas de simulación de movimiento están basadas en estructuras de cadenas cinemáticas cerradas, típicas de los robots paralelos. El movimiento de la plataforma de simulación está dado por los grados de libertad (GDL) con que consta el sistema.

Uno de los aspectos importantes en el diseño y control de un robot paralelo es la obtención del modelo dinámico del mismo, lo cual posibilita implementar técnicas de control requeridas para gobernar el sistema y lograr el posicionamiento deseado de la 
plataforma móvil en el espacio cartesiano. Existen numerosos obstáculos en la obtención del modelo dinámico, entre ellos, la aparición de parámetros desconocidos, el efecto de las interacciones dinámicas, así como otros aspectos difíciles de estimar como las fuerzas de fricción, variaciones de carga, etc. [1]. Algunos autores dejan de considerar el sistema completo desde el punto de vista dinámico, considerando que cada actuador puede ser controlado independientemente con leyes de control más robustas que el simple PID [2]. Un control basado en modelo dinámico exige alta calidad para el modelo obtenido, aspecto difícil de lograr en la práctica por los elementos antes mencionados, por ello frecuentemente se efectúan aproximaciones con el fin de obtener un modelo más práctico, fácil de implementar y que cumpla con las exigencias de tiempo real asociadas a este tipo de sistemas.

No obstante, para lograr el control es imprescindible un modelo dinámico que se acerque lo más fielmente posible al comportamiento real del sistema mecánico. Modelo dinámico que además sea factible de implementar, simular y que permita diseñar una estrategia de control adecuada basada en dicho modelo.

Este trabajo, no se presenta una dinámica con alto nivel de exactitud sino simplificada, para lograr así una menor complejidad en la implementación y diseño del controlador.

No son pocas las formulaciones que clásicamente se usan para la realización del modelo dinámico. Entre ellas podemos mencionar el clásico método de Newton-Euler [3], el método de Lagrange-Euler basado en el balance de energía [4], las ecuaciones de Gibbs-Appell [5], y el principio del Trabajo Virtual [6], muy empleado en aplicaciones de tiempo real.

El más común de los métodos basados en vectores es la aproximación de Newton-Euler, donde cada cuerpo rígido de un sistema mecánico es cortado libre y las ecuaciones dinámicas son derivadas al solucionar balances de fuerzas y torques. Para robots de tipo serie su formulación recursiva es muy efectiva, pero en el caso de los robots paralelos la formulación no-recursiva del método de Newton-Euler es más apropiada [7]. Una ventaja importante del método Newton-Euler es la forma lineal de los parámetros del sistema dinámico de modo que permite el uso de estimadores lineales en el proceso de identificación [8].

En la formulación de Newton-Euler, las fuerzas y torques de reacción entre todas las partes móviles, incluyendo las demandas de los actuadores pueden ser fácilmente calculadas, proveyendo un modelo excelente para análisis y estudios de la estructura mecánica. Debido al alto número de ecuaciones y su gran complejidad, la eficiencia computacional del modelo puede ser considerada pobre, haciéndolo difícil de aplicar en control de tiempo real cuando el mecanismo cuenta con más de tres GDL [9].

Una vez obtenido el modelo dinámico del robot se puede entonces pasar al diseño del control. El control dinámico tiene por misión procurar que las trayectorias articulares realmente seguidas por el robot $q(t)$ sean lo más parecidas posibles a las deseadas $q_{d}(t)$, lo cual tiene relación con el control desde el punto de vista cinemático. Este mismo análisis se puede efectuar en el espacio cartesiano, donde entonces el vector $x(t)$ debe seguir al vector deseado $x_{d}(t)$, minimizando el error espacial de posicionamiento de la plataforma móvil [10]. Para ello hace uso del conocimiento del modelo dinámico del robot y de las herramientas de análisis y diseño aportadas por la teoría de control [11].

El control por dinámica inversa es una herramienta valiosa que puede producir un buen desempeño del sistema [12][13], mejor aun cuando el modelo completo del sistema es usado con mayor exactitud en los parámetros. No obstante, siempre existirán incertidumbres no consideradas en el modelo dinámico debido a la dificultad de identificar con precisión los parámetros del modelo, o por naturales simplificaciones asumidas al modelar [14]. Por lo tanto, el desempeño ideal del método de control por dinámica inversa suele degradarse. Para resolver este problema se hace necesario introducir otra estrategia de control que trabaje a la par, en este caso un PD [15][16].

En este artículo se presenta el procedimiento de modelado dinámico seguido para describir el comportamiento de un simulador de conducción de 2 GDL accionado por cilindros neumáticos, se plantea el control del mismo para lograr el adecuado posicionamiento de la plataforma móvil, según las exigencias relativas a la aplicación de simulador industrial de movimiento, para finalmente simular los resultados derivados de la estrategia de control implementada.

\section{DESCRIPCIÓN DE LA PLATAFORMA SIMPRO DE 2 GDL}

El objeto de aplicación de este trabajo se corresponde con una plataforma de dos GDL producida en el Centro de Investigación y Desarrollo de Simuladores "CIDSIM", la misma permite la realización de dos movimientos rotacionales sobre los ejes $(\mathrm{x}, \mathrm{y})$, denominados cabeceo y alabeo respectivamente, y se logran mediante el accionar de dos cilindros neumáticos (actuadores lineales) que conforman las cadenas cinemáticas cerradas que unen la base fija con la plataforma móvil .

El simulador de marca SIMPRO está compuesto por una cabina de conducción con los mandos reales que simulan el comportamiento al cual se enfrenta el conductor y un monitor para visualizar el entorno virtual. La Tabla 1 resume algunos datos y principales especificaciones del simulador de conducción. Se considera que la masa total de la cabina incluye el propio peso de la cabina y del conductor cuya masa se estima en $70 \mathrm{Kg}$.

Tabla 1: Datos Mecánicos del Simulador.

\begin{tabular}{|c|c|}
\hline Parámetro & Valor \\
\hline Masa total de la cabina & $500 \mathrm{Kg}$ \\
\hline Posición centro de masa $(\mathrm{CM})(\mathrm{x}, \mathrm{y}, \mathrm{z})$ & $(0,0,700) \mathrm{mm}$ \\
\hline Distancia del origen a cada cilindro & $560 \mathrm{~mm}$ \\
\hline Elongación de los cilindros & $\pm 150 \mathrm{~mm}$ \\
\hline Ángulos de ladeo & $-13^{\circ}$ a $19^{\circ}$ \\
\hline Ángulos de cabeceo & $13^{\circ} \mathrm{a}-19^{\circ}$ \\
\hline Aceleración máx. actuadores & $1000 \mathrm{~mm} / \mathrm{s}^{2}$ \\
\hline
\end{tabular}

Fuente: Autores, (2010).

La base móvil sobre la cual se coloca la cabina conducción pivotea sobre una columna central mediante una articulación universal pasiva, ver Figura 1. El movimiento de la plataforma móvil se logra mediante la acción de dos cilindros FESTO DNC100-400 de doble efecto, cuyos desplazamientos son gobernados por una válvula solenoide proporcional de flujo MPYE-5-3/8. 


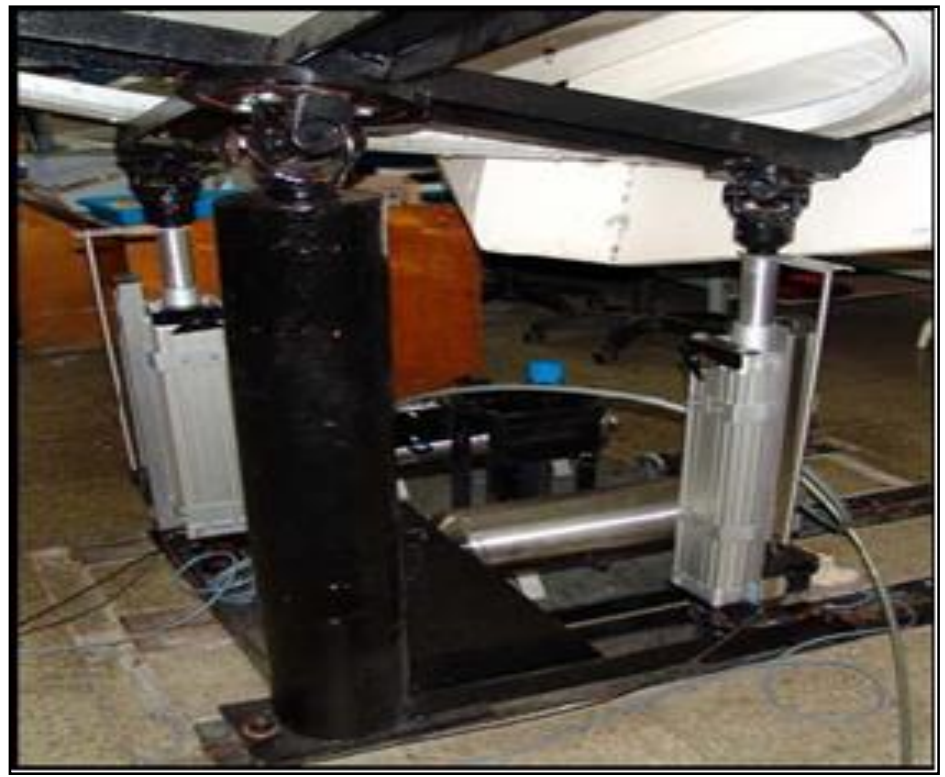

Figura 1: Simulador industrial de conducción SIMPRO de 2 GDL. Fuente: Autores, (2010).

En los extremos de las articulaciones prismáticas, se encuentran uniones universales que permiten lograr los grados de movilidad necesarios para obtener las orientaciones de la plataforma móvil. Los desplazamientos lineales de los cilindros se traducen en el elemento final en rotaciones alrededor de los ejes $\left(\mathrm{x}^{\prime}, \mathrm{y}^{\prime}\right)$ del sistema de referencia móvil, las cuales producen en el mismo variaciones en el ángulo de cabeceo (rotación respecto al eje $x^{\prime}$ ) y ángulo de alabeo (rotación respecto al eje y'); gracias a lo cual se pueden simular las pendientes del mundo virtual que a su vez son visualizadas en un monitor [17].

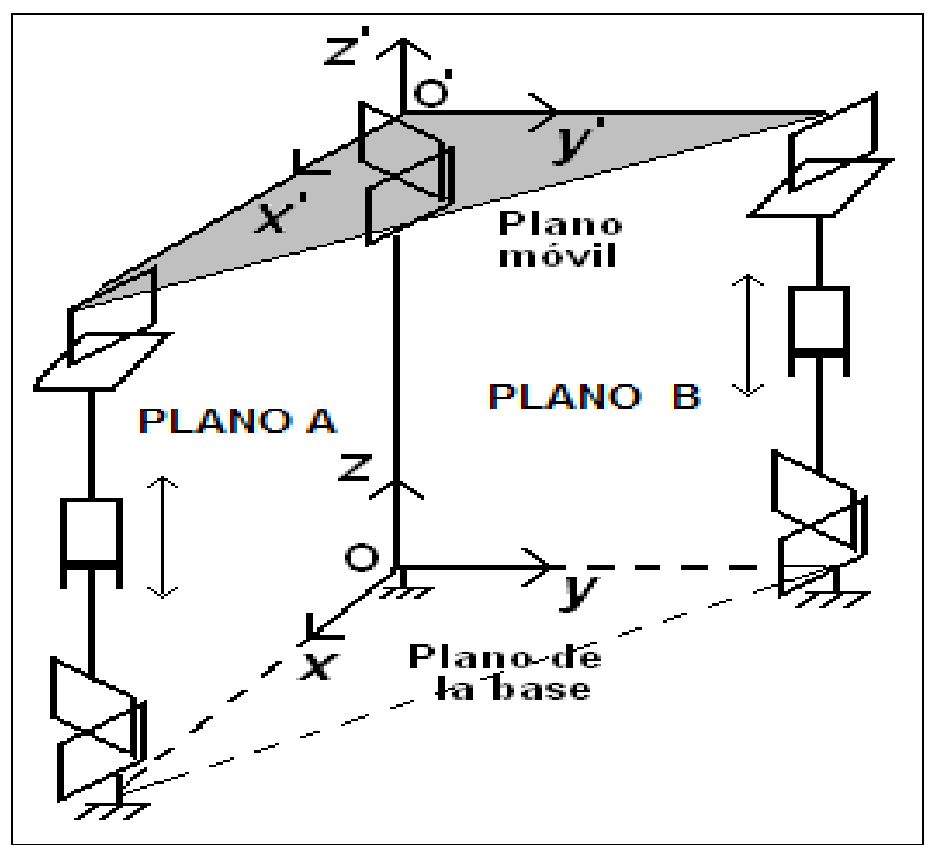

Figura 2: Orientaciones del Vehículo en el Espacio.

Fuente: Autores, (2010).

Como premisa para la obtención del modelo dinámico resulta necesario disponer de las relaciones cinemáticas que caracterizan el robot. Para el caso que nos ocupa, las expresiones cinemáticas de este sistema se obtienen considerando trabajos previos publicados, donde se modela y simula el comportamiento cinemático-dinámico de este robot [18]. No obstante el modelo dinámico aquí planteado parte de evaluar las expresiones de la cinemática diferencial, considera el sistema desacoplado, y además se expresa en el espacio articular. Por consiguiente no resulta práctico a la hora de implementar seguimiento de trayectoria.

\section{MODELO DINÁMICO DE LA PLATAFORMA}

La aproximación dinámica se obtendrá mediante el empleo del método Newton-Euler. Para ello se considera que toda la massa de la cabina está contenida en el centro de masa, ubicado a $0,5 m$ del punto de giro sobre el pedestal, distancia definida por la variable $\mathrm{CM}$, donde se define $l$ como la longitud del punto de giro al punto en que se ejerce la fuerza.

La ecuación en el espacio articular es como sigue:

$$
f=M(q) \ddot{q}+C(q, \dot{q}) \dot{q}+g(q)
$$

Donde:

$$
\begin{aligned}
& f=\left[\begin{array}{l}
F_{1} \\
F_{2}
\end{array}\right]: \text { Fuerza aplicada por los cilindros neumáticos. } \\
& q=\left[\begin{array}{l}
d_{1} \\
d_{2}
\end{array}\right]: \text { Desplazamiento de los cilindros neumáticos. }
\end{aligned}
$$

En el espacio de tareas la ecuación (1) queda de la forma:

$$
f_{x}=M_{x}(x) \ddot{x}+C_{x}(x, \dot{x}) \dot{x}+G(x)
$$

Donde:

$$
\begin{aligned}
f_{x} & =\left[\begin{array}{l}
\tau_{1} \\
\tau_{2}
\end{array}\right]: \text { Momento producido por cada cilindro. } \\
x & =\left[\begin{array}{l}
\alpha \\
\sigma
\end{array}\right]: \text { Desplazamiento angular producido por cada }
\end{aligned}
$$

cilindro, siendo $\alpha$ y $\sigma$ los ángulos de rotación en los planos A y $\mathrm{B}$ respectivamente.

Se considerará que la velocidad de desplazamiento del sistema es baja, por tanto:

$$
C_{x}(x, \dot{x})=C(q, \dot{q})=0
$$

Para valores pequeños de $\alpha$ y $\sigma$ se cumplirá:

$$
d_{1}=l \alpha \text { y } d_{2}=l \sigma
$$
queda:

por tanto, la matriz Jacobiana simplificada del sistema

$$
J=\left[\begin{array}{ll}
l & 0 \\
0 & l
\end{array}\right]
$$




\section{III.1. ANÁLISIS DESACOPLADO}

El análisis desacoplado se desarrolla sin considerar interacciones entre las fuerzas de los actuadores como se indica en la Figura 3 (a) y (b).

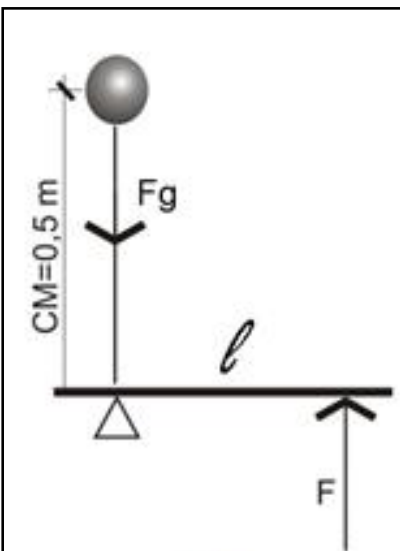

(a)

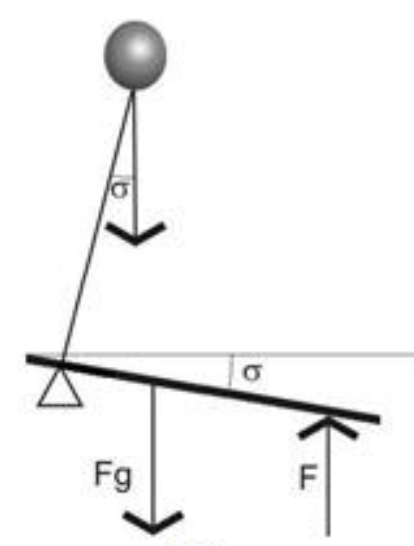

(b)
Figura 3: Vista perpendicular al Plano B (a) con masa concentrada en el centro de masa (b) girada con ángulo $\sigma$.

Fuente: Autores, (2015).

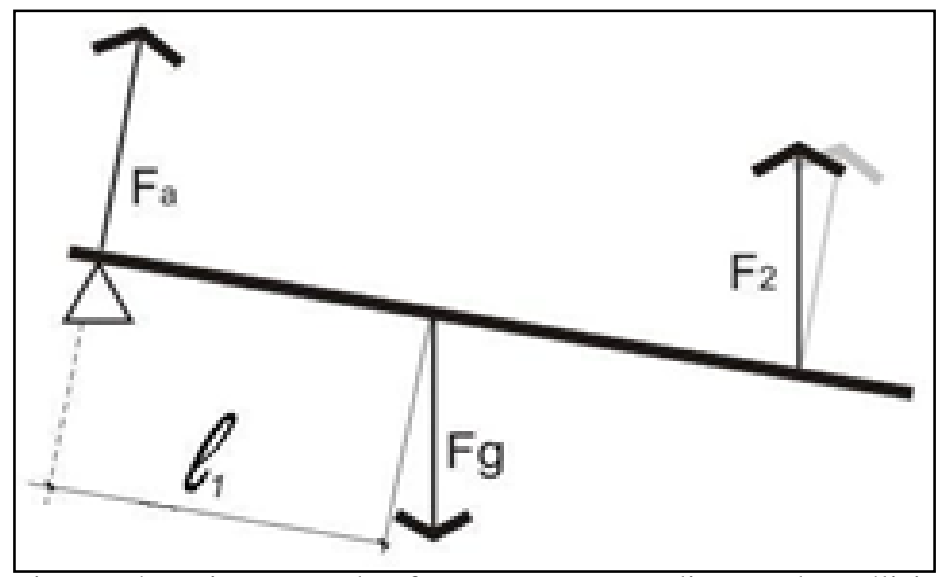

Figura 4: Diagrama de fuerzas correspondiente al análisis desacoplado.

Fuente: Autores, (2015).

$l_{1}$ : Distancia del punto de giro al punto en que se ejerce la fuerza de gravedad.

$F_{2}$ : Fuerza ejercida por el cilindro 2 .

$F_{a}:$ Fuerza del sistema sobre el apoyo.

$F_{g}:$ Fuerza de gravedad.

$$
\begin{gathered}
l_{1}=C M \tan \sigma \\
F_{a}+F_{2} \cos \sigma=m g \cos \sigma \\
m g l_{1} \cos \sigma-F_{2} l \cos \sigma=0 \\
m g l_{1}-F_{2} l=0
\end{gathered}
$$

$$
\begin{gathered}
F_{2}=\frac{m g C M \tan \sigma}{l} \\
\tau_{2}=m g C M \tan \sigma \\
F_{1}=\frac{m g C M \tan \alpha}{l} \\
\tau_{1}=m g C M \tan \alpha
\end{gathered}
$$

\section{III.2. ANÁLISIS ACOPLADO}

Al desarrollar el análisis acoplado del sistema se deba considerar primero el caso en el cual $\alpha=\sigma=0$, donde $\tau_{1}=\tau_{2}=0$ y por consiguiente no hay giro en ninguno de los planos.

Mientras que, al girar $\alpha$ sin ejercer fuerza en el punto "b" por parte del cilindro 2 se llega al análisis de la Figura 5 (b) donde la distancia del centro de masa al punto de apoyo se ve afectada por el factor $\cos \alpha$.

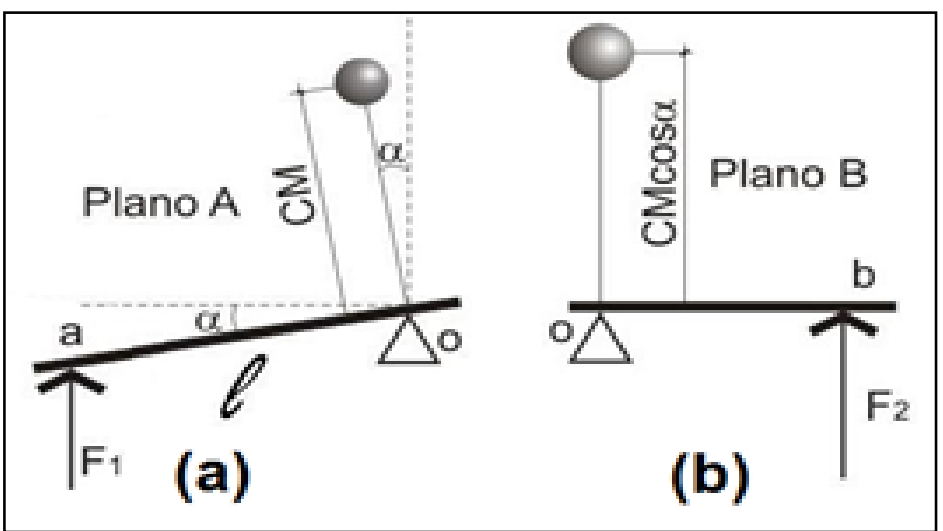

Figura 5: (a) Vista perpendicular al Plano A con ángulo $\alpha$ (b) Vista perpendicular al Plano B con ángulo $\alpha$.

Fuente: Autores, (2015).

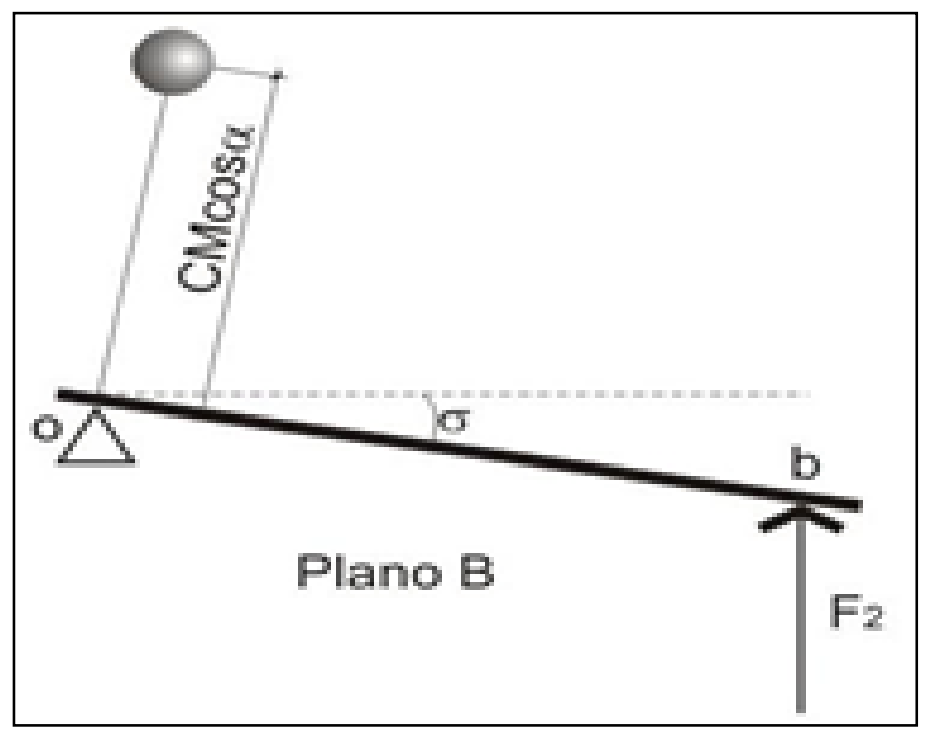

Figura 6: Vista perpendicular al Plano B girados los ángulos $\sigma$ y $\alpha$. Fuente: Autores, (2015). 
Entonces aplicando fuerza en el punto "b" con el ángulo $\alpha$ girado como muestra la Figura 6 y considerando las ecuaciones desacopladas se puede llegar al siguiente desarrollo:

$$
F_{2}=\frac{m g C M \cos \alpha \tan \sigma}{l}
$$

Donde $\tau_{2}$ queda definido por:

$$
\tau_{2}=m g C M \cos \alpha \tan \sigma
$$

Por simetría:

$$
\begin{gathered}
F_{1}=\frac{m g C M \cos \sigma \tan \alpha}{l} \\
\tau_{1}=m g C M \cos \sigma \tan \alpha
\end{gathered}
$$

La fuerza aplicada en el punto "a" de la Figura 5 (a) hace girar el sistema alrededor del eje "ob", por tanto el término de aceleración por $\alpha$ sería $I_{0 b} \ddot{\alpha}$; donde $I_{0 b}$ es el momento de inercia del sistema alrededor del eje "ob".

$$
I_{o b}=m C M^{2}
$$

Por simetría el término de aceleración por $\sigma$ sería $I_{o a} \ddot{\sigma}$ entonces:

$$
I_{o a}=m C M^{2}
$$

Sobre esta base la ecuación dinámica del sistema queda:

$$
\left[\begin{array}{l}
\tau_{1} \\
\tau_{2}
\end{array}\right]=\left[\begin{array}{cc}
m C M^{2} & 0 \\
0 & m C M^{2}
\end{array}\right]\left[\begin{array}{l}
\ddot{\alpha} \\
\ddot{\sigma}
\end{array}\right]+\left[\begin{array}{l}
m g \cos \sigma \tan \alpha \\
m g \cos \alpha \tan \sigma
\end{array}\right]
$$

Para valores pequeños de $\alpha$ y $\sigma$ donde $\operatorname{sen} \alpha \approx \alpha$ con $\cos \alpha \approx 1$ y $\operatorname{sen} \sigma \approx \sigma$ con $\cos \sigma \approx 1$, el sistema linealizado queda:

$$
\left[\begin{array}{l}
\tau_{1} \\
\tau_{2}
\end{array}\right]=\left[\begin{array}{cc}
m C M^{2} & 0 \\
0 & m C M^{2}
\end{array}\right]\left[\begin{array}{l}
\ddot{\alpha} \\
\ddot{\sigma}
\end{array}\right]+\left[\begin{array}{l}
m g \\
m g
\end{array}\right]\left[\begin{array}{l}
\alpha \\
\sigma
\end{array}\right]
$$

\section{APLICACIÓN DE LA ESTRATEGIA DE CONTROL}

El control dinámico en el espacio de tareas utilizando el método de Control por Modelo Dinámico Inverso, para la plataforma de dos grados de libertad se describe a continuación.

Primeramente, sería útil reescribir la ecuación del modelo dinámico en el espacio de tareas como se presenta en (2):

$$
\tau=M_{x}(x) \ddot{x}+C_{x}(x, \dot{x}) \dot{x}+G(x)
$$

Puesto que se desprecian las fuerzas Centrípetas y de Coriolis, las cuales conforman la matriz $C_{x}$, la ecuación del modelo ahora queda:

$$
\tau=M_{x}(x) \ddot{x}+G_{x}(x)
$$

Esta ecuación a su vez puede ser escrita de la siguiente forma:

$$
\tau=M_{x}(x) u+G_{x}(x)
$$

donde $u$ es el vector de entrada al lazo interno de control (teniendo en cuenta que el esquema presenta la forma lazo interno/lazo externo) y tiene unidades de aceleración. Se asume que no existen disturbios, fuerzas externas que afecten el desempeño del sistema. Esto nos lleva a un esquema de control como el que se muestra en la Figura 7 donde se implementa la estrategia de Control por Modelo Dinámico Inverso acompañado de un controlador PD.

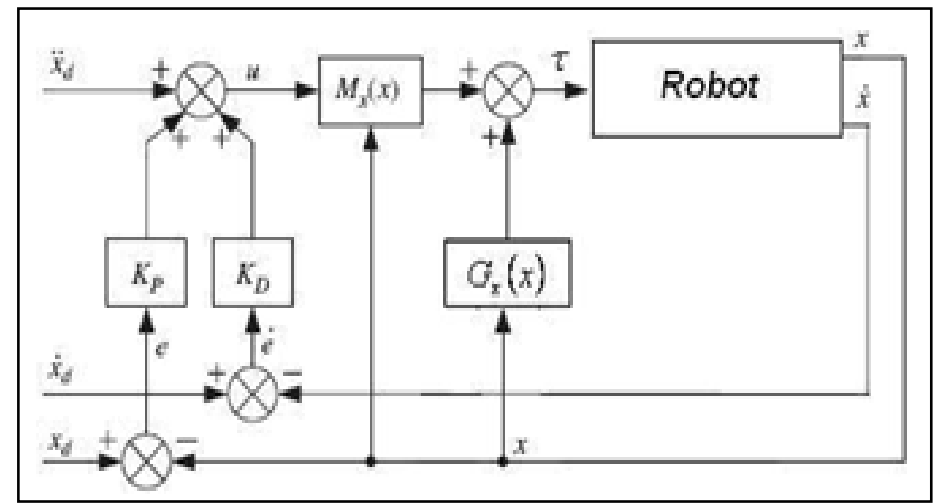

Figura 7: Diagrama en bloques del Esquema de Control por Modelo Dinámico Inverso.

Fuente: Autores, (2017).

Siendo $x_{d}$ la trayectoria deseada para la plataforma móvil, $K_{P}$ y $K_{D}$ ganancias del controlador.

Con $\ddot{x}=u$ entonces la señal de referencia $\mathrm{r}$ se define por:

$$
r=\ddot{x}_{d}+K_{D} \dot{x}_{d}+K_{P} x_{d}
$$

De esta forma el vector de entrada al lazo interno de control será:

$$
u=\ddot{x}_{d}+K_{D}\left(\dot{x}_{d}-\dot{x}\right)+K_{P}\left(x_{d}-x\right)
$$

siendo $x$ la trayectoria real seguida por la plataforma móvil.

Sustituyendo (23) en $\ddot{x}=u$ se obtiene la ecuación diferencial de segundo orden del error:

$$
\ddot{e}+K_{D} \dot{e}+K_{P} e=0
$$

Donde $e=x_{d}-x$ es el error de desplazamiento en la trayectoria. 
En el diagrama anterior Figura 9 se muestra claramente que el método de Control por Modelo Dinámico Inverso se encuentra acompañado de un controlador Proporcional-Derivativo (PD) cuyas ganancias KP y KD deben ser sintonizadas considerando que [19][3]:

$$
\begin{gathered}
K_{P}=\operatorname{diag}\left(K_{P}\right)=\operatorname{diag}\left(w_{n}^{2}\right) \\
K_{D}=\operatorname{diag}\left(K_{D}\right)=\operatorname{diag}\left(2 \zeta w_{n}\right)
\end{gathered}
$$

Dado que el control se diseña para una estructura mecánica es conveniente hacer la respuesta críticamente amortiguada, para lo cual se fija la razón de amortiguamiento $\zeta=1$, eliminando así sobrecrestas indeseadas. Más aun, para evitar que el sistema caiga en resonancia y oscilaciones estructurales existe el criterio de definir la frecuencia natural $\left(w_{n}\right)$ nunca mayor a la mitad de la frecuencia de resonancia estructural $\left(w_{s}\right)$ del mecanismo paralelo [15].

Tomando que $w_{s}$ es $6 \mathrm{~Hz}$ entonces $w_{n}$ sería:

$$
w_{n}=2 \pi \times 3 \mathrm{~Hz}=18.85 \mathrm{rad} / \mathrm{s}
$$

Así se pueden sintonizar $K_{P}$ y $K_{D}$ como:

$$
\begin{aligned}
& K_{P}=355.3 \\
& K_{D}=37.7
\end{aligned}
$$

\section{SIMULACIÓN}

Se desarrolló en MATLAB el diagrama de bloques del sistema y se simuló su respuesta ante una trayectoria prefijada, la función seno.

El diagrama de la Figura 7 se implementó en Simulink con el fin de simular la respuesta del sistema y el error ante seguimiento de trayectoria.

La señal de entrada es la función seno con amplitud de 0.5 y a una frecuencia de $0.1 \mathrm{rad} / \mathrm{seg}$. El diagrama en Simulink se muestra en la Figura 8.

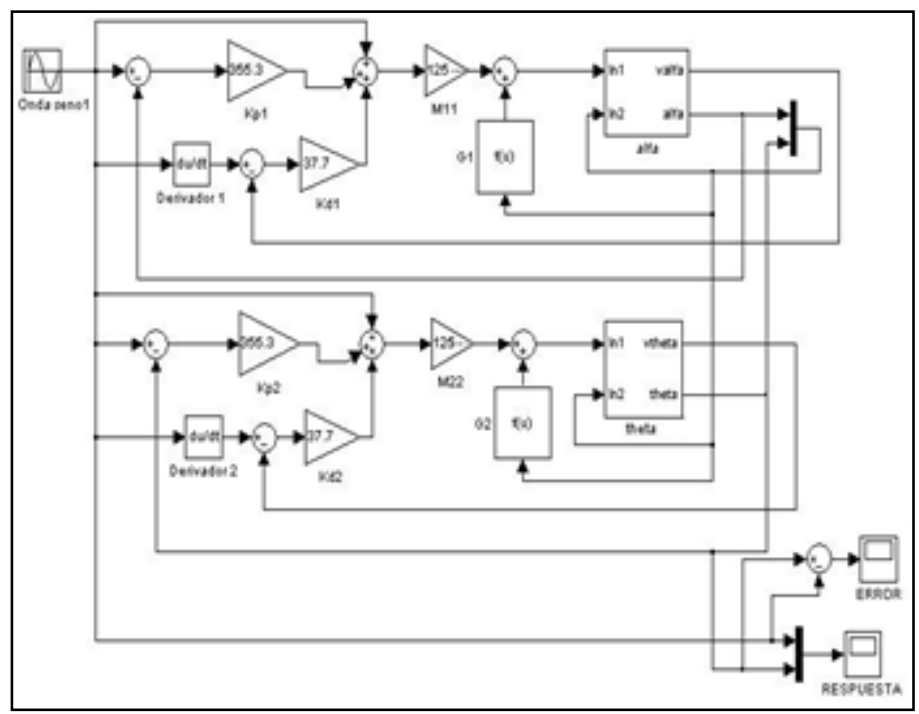

Figura 8: Diagrama de bloques en Simulik.

Fuente: Autores, (2017).
Los resultados de la simulación se muestran en las Figura 9 y Fig. 10.

Se puede apreciar en estas figuras la pequeñez del error que, al sintonizar de esta forma las ganancias, ocurre en el seguimiento de trayectoria.

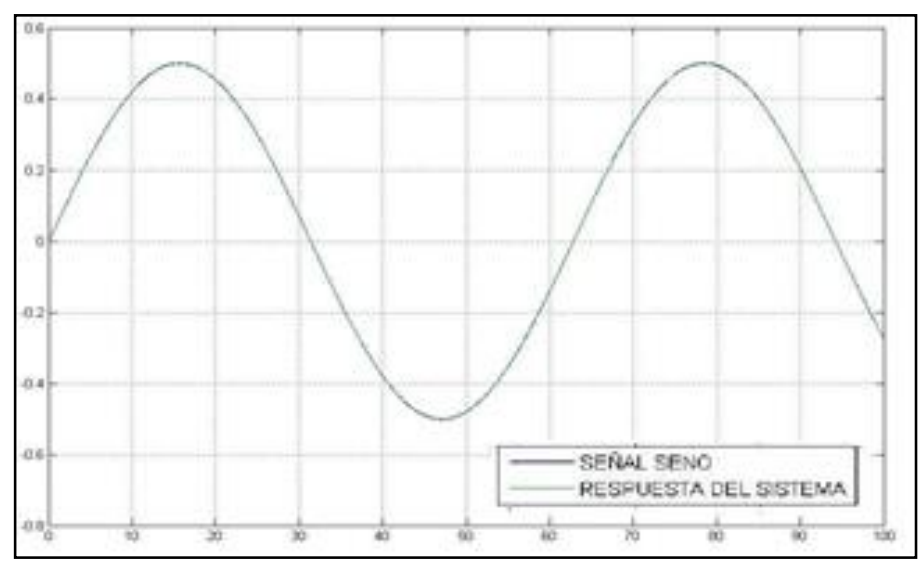

Figura 9: Respuesta del sistema contra la trayectoria deseada. Fuente: Autores, (2017).

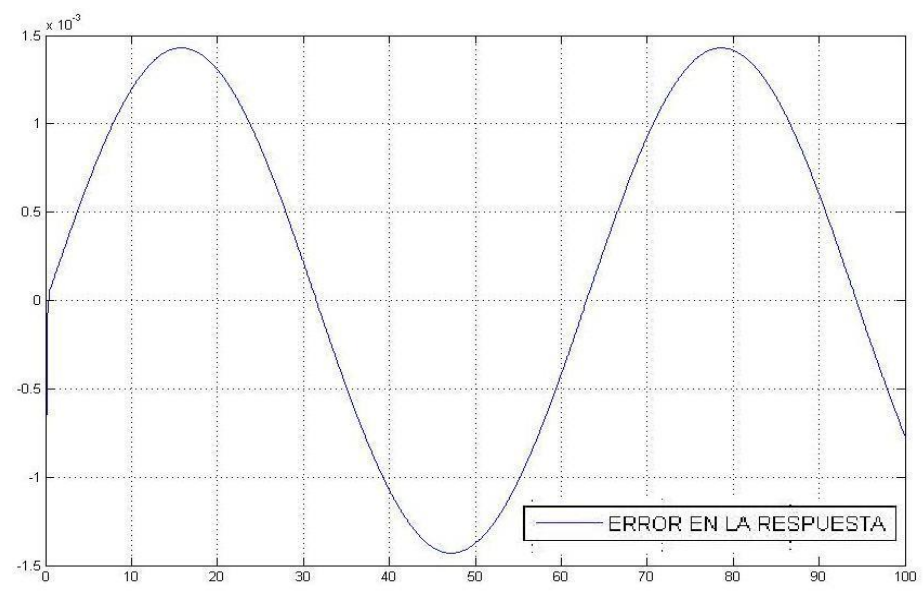

Figura 10: Gráfica del error.

Fuente: Autores, (2017).

Como se aprecia en las Figura 9 y Figura 10 el sistema controlado describe un seguimiento de trayectoria, con muy pequeño error, del orden de $1.5 \times 10^{-3}$ metros, lo cual es totalmente imperceptible para los sentidos humanos cuando se manipula la plataforma de simulación.

\section{CONCLUSIONES}

El modelo dinámico del sistema juega un papel fundamental en el buen desempeño del control. En la medida que el modelo represente el comportamiento dinámico del robot así será la exactitud con que se logrará controlar y la respuesta que se obtendrá.

A su vez el control en el espacio de tareas, o espacio cartesiano, resulta eficaz en mecanismos paralelos como el que se ha tratado. Esto es así siempre que se cuente con un sistema de medición rápido y efectivo que brinde la información de las variables que definen la pose de la plataforma.

La simulación del sistema controlado permite conocer de antemano el comportamiento del mecanismo sin la necesidad de implementar el sistema de control físico evitando con ello 
posibles daños en la estructura mecánica del robot durante las pruebas experimentales.

\section{REFERÉNCIAS}

[1] K. M. Chen Zhengsheng, Liu Ming and You Wei, "Dynamic Modelling and Trajectory Tracking of Parallel Manipulator with Flexible Link," International Journal of Advanced Robotic Systems, vol. 10, pp. 328-337, 2013.

[2] J.-P. Merlet, Parallel Robots, 2 ed. France: Springer Editorial, 2006.

[3] S. H. Mark W. Spong, and M. Vidyasagar, Robot Dynamics and Control, 2 ed., 2004.

[4] B. H. Houssem Abdellatif, "Computational efficient inverse dynamics of 6-DOF fully parallel manipulators by using the Lagrangian formalism," Science Direct, vol. 44, pp. 192-207, 2008.

[5] S. P. V. Mata, J.L. Cuadrado and F. Valero "Inverse dynamic problem in robots using Gibbs-Appell equations," Robotica, vol. 20, pp. 59-67, 2002.

[6] R. C. Limón, "Modelo Matemático de un Robot Paralelo de Seis Grados de Libertad," Ph. D. Thesis, Computer, Electronics, Physics and Innovation, University of Americas, Puebla, 2006.

[7] L.-W. Tsai, Robot analysis: the mechanics of serial and parallel manipulators, 1 ed. United States of America: John Wiley \& Sons, INC, 1999.

[8] P. A. M. Jens Kroneis, Steven Liu, "Dynamic Modeling and Identification of a Complex-structured Parallel Robot," in 17th World Congress The International Federation of Automatic Control, 2008, p. 6.

[9] S. C. T. F. E. L. L. Cabral, "Dynamics and Jacobian Analysis of parallel architecture robot: The Hexa," ABCM Symposium Series in Mechatronics, vol. 2, pp. 166173, 2006.

[10] V. V. Yogesh Singh, M. Santhakumar, , "Dynamic Modelling and Control of a 3-DOF Planar Parallel Robotic (XY0Z Motion) Platform," presented at the International Conference on Advances in Manufacturing and Materials Engineering, ICAMME 2014, 2014.

[11] L. F. P. Antonio Barrientos, Carlos Balaguer, Rafael Aracil, Fundamentos de Robótica, 1 ed. España: McGrawHill/Interamaricana, 1997.

[12] W. a. D. Khalil, E. , "Modeling, identification and control of robots," Hermes Penton Science, 2002.

[13] B. C. Santhakumar Mohan, "Inverse dynamics and trajectory tracking control of a new six degrees of freedom spatial 3-RPRS parallel manipulator," Mechanical Sciences, vol. 8, pp. 235-248, 2017.

[14] M. K. Mohamadreza Homayounzad, Mostafa Ghobad, "A Robust Tracking Controller for Electrically Driven
Robot Manipulators: Stability Analysis and Experiment," International Journal of Automation and Computing, vol. 12, pp. 83-92, 2015.

[15] Q. X. Yangmin Li, "Dynamic modeling and robust control of a 3-PRC translational parallel kinematic machine. ," Robotics and Computer-Integrated Manufacturing, Elsevier, 2008.

[16] S. C. Weiwei Shang, "Nonlinear computed torque control for a high-speed planar parallel manipulator," Mechatronics, vol. 19, pp. 987-992, 2009.

[17] E. R. Eduardo Izaguirre, Sandor E. Dominquez, "Modelo Cinemático y Dinámico para Robot Paralelo de Dos Grados de Libertad," in Conferencia Internacional de Ingeniería Eléctrica, 2008.

[18] E. I. C. Orlando Urquijo Pascual, Luis Hernández Santana, "Control de trayectoria en el espacio cartesiano de robot paralelo de 2GDL usando modelo cinemático vectorial," Revista de Ingeniería Electrónica, Automática y Comunicaciones RIELAC, vol. 38, pp. 72-82, Mayo Agosto 2017.

[19] V. S. a. A. L. R. Kelly, Control of Robot Manipulators in Joint Space, 1 ed. ed. Germany: Springer, 2005. 